\title{
Ultrasound imaging for bladder hernia: a rare cause of female inguinal hernia
}

\author{
Ke-Vin Chang ${ }^{1,2}$, Wei-Ting Wu ${ }^{1}$, Levent Özçakar ${ }^{3}$
}

${ }^{1}$ Department of Physical Medicine and Rehabilitation, National Taiwan University Hospital, Bei-Hu Branch and National Taiwan University College of Medicine, Taipei, Taiwan, ${ }^{2}$ Center for Regional Anesthesia and Pain Medicine, Wang-Fang Hospital, Taipei, Medical University, Taipei, Taiwan, ${ }^{3}$ Department of Physical and Rehabilitation Medicine, Hacettepe University Medical School, Ankara, Turkey

\section{To the Editor,}

A 46-year-old woman had a painless mass over her left inguinal area for the last six months. She initially visited the outpatient clinic of general surgery where inguinal hernia was suspected and was then sent for ultrasound (US) examination. The transducer was first placed along the inguinal fold medial to the external iliac artery. No bowel content was recognized besides the round ligament. However, an anechoic compressible cyst with a reversed funnel shape was visualized (fig 1A). A tract extending from the tip of the funnel toward the pubic bone was also identified. The transducer was then redirected to the sagittal plane, disclosing the bladder cranial to the cystic mass (fig 1B). Dynamic compression of the bladder using the transducer elicited a change in the cyst size (Video 1, on the journal site). Under the impression of bladder hernia, she was referred back for surgical repair.

Compared with men, women are less likely to develop inguinal hernia, whereby the lifelong risk has been reported to range between 3-5.8\% [1]. Female hernia can be mainly divided into the direct type (caused by the weak abdominal wall) and indirect type (derived from bowel contents sliding along the inguinal canal). The former type is less prevalent [2]. Bladder hernia can be classified within the indirect type of inguinal hernia and is usually manifested by part of the bladder being herniated into the inguinal canal. Bladder hernia is relatively rare,

Received 22.07.2021 Accepted 05.10.2021

Med Ultrason

2021, Vol. 23, No 4, 493-494, DOI: 10.11152/mu-3385,

Corresponding author: Ke-Vin Chang, MD, PhD

Department of Physical Medicine and Rehabilitation, National Taiwan University Hospital, Bei-Hu Branch and National Taiwan University College of Medicine, Taipei, Taiwan E-mail: kvchang011@gmail.com with a prevalence of $1-3 \%$ of all types of inguinal hernia [3]. Most patients are asymptomatic, although dysuria, urgency and nocturia could be the most common findings in symptomatic cases. The diagnostic imaging tools encompass excretory urography, retrograde cystography, computed tomography, US and magnetic resonance imaging. Under US examination, the herniated portion of the bladder is seen as a fluid-filled sac, emerging from the medial aspect of the deep inguinal ring. The deep inguinal ring can be identified by locating the merging point of the inferior epigastric artery with the external iliac artery. A connecting tract between the herniated sac

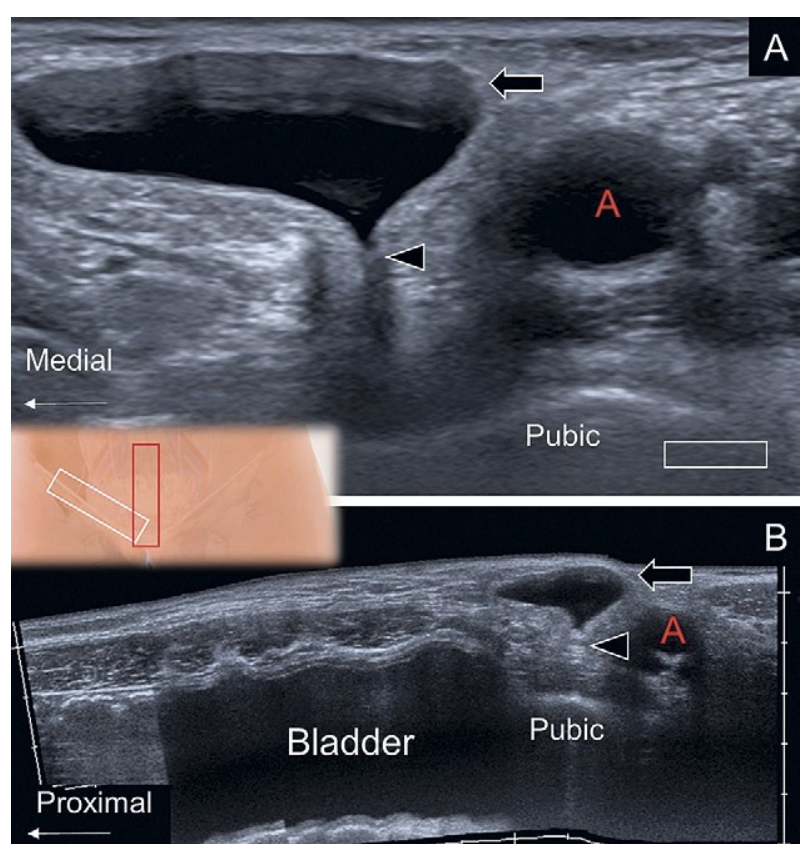

Fig 1. Ultrasound imaging of bladder hernia (arrows) in the horizontal (A) and sagittal panoramic (B) views. Arrowheads indicate the connecting tract between the herniated sac and the bladder. A, external iliac artery. 
and bladder may be found. Dynamic testing by compressing and decompressing the bladder to visualize the changing size of the cyst may be helpful for diagnosis. In short, the present case highlights the usefulness of static and dynamic US imaging in the diagnosis of bladder hernia during daily clinical practice where US is actually used as the extension of routine musculoskeletal examination.

Acknowledgment: This study was made possible by (1) the Ministry of Science and Technology (MOST 106-2314-B-002-180-MY3, 109-2314-B-002-114-MY3, and 109-2314-B-002-127), and (2) the Taiwan Society of

Ultrasound in Medicine.

\section{References}

1. Köckerling F, Koch A, Lorenz R. Groin Hernias in WomenA Review of the Literature. Front Surg 2019;6:4-4.

2. Chawla S. Inguinal hernia in females. Med J Armed Forces India 2001;57:306-308.

3. Bacigalupo LE, Bertolotto M, Barbiera F, et al. Imaging of Urinary Bladder Hernias. AJR Am J Roentgenol 2005; 184:546-551.

\title{
Rare hand mass: malignant peripheral nerve sheath tumor
}

\author{
Miao Shi ${ }^{1}$, Xin Li ${ }^{1,2}$, Xiaojie Pan ${ }^{1}$, Liangliang Wang ${ }^{1}$, Caiying Pei ${ }^{1}$, Liqian Sun ${ }^{1}$, Qinghua Zhao ${ }^{1}$
}

${ }^{1}$ Department of Ultrasound, Liaocheng People's Hospital, Liaocheng, ${ }^{2}$ Shandong First Medical University, Taian, Shandong, China

\section{To the Editor,}

A 28-year-old female was presented with a 4-year history of mass on her right palm (fig 1a). The mass was soft, cystic and painless. The surrounding skin was not reddened and swollen, and the distal end of the limb felt normal. It had progressively enlarged recently and the size was $3 \times 3 \times 1.5 \mathrm{c} \mathrm{m}$. A mass in the right axillary area was found 3 months ago, about $2.3 \times 1.9 \times 1.2 \mathrm{~cm}$ in size, which was tactile similar to the mass on the hand. Ultrasound examination showed that those masses were uneven and hypoechoic with a rich blood flow signal (fig 1b-d). After the surgery, it was considered to be malignant peripheral nerve sheath tumor (MPNST) by immunohistochemistry and the axillary mass was lymph node metastasis (fig 1e,f).

MPNST originates from the Schwann cells and has the potential of neuronal differentiation. It can occur

Received 18.07.2021 Accepted 05.10.2021

Med Ultrason

2021, Vol. 23, No 4, 494-495, DOI: 10.11152/mu-3418,

Corresponding author: Qinghua Zhao

Department of Ultrasound, Liaocheng

People's Hospital, 67 Dongchang West Road,

Dongchangfu District,

Liaocheng 252000, Shandong, China

Phone: 0635-8272175

E-mail: zqh8512@126.com in any part of the body that contains peripheral nerve branches or nerve sheath. It is rare in the palm of the hand. It is a tumor that grows slowly at the beginning, then grows suddenly and rapidly [1]. The tumor is mostly painless and it can also produce symptoms such as motor and sensory dysfunction caused by nerve compression [2]. MPNST is highly aggressive and can be locally invaded. It is prone to distant lymph node metastasis and hematogenous metastasis [3] and it is easy to recur after surgery. S-100 and SOX10 are sensitive markers [4].

On ultrasound, the tumor mostly shows a large hypoechoic mass, no capsule or incomplete capsule and uneven internal echo. Additionally, CDFI shows rich blood flow signals. Ultrasound rarely shows that it is connected to the nerve directly, which is different from the benign schwannoma's characteristic of connecting to the nerve. In this case, there was no relationship between the tumor and nerve, which may be related to the fact that the tumor originated from the smaller nerve and the normal nerve structure has been destroyed.

In conclusion, ultrasound examination can provide information such as tumor size, echo characteristics, blood supply and infiltration of surrounding tissues, so as to provide evidence for clinical diagnosis. The possibility of this disease should be considered in patients with similar manifestations. 


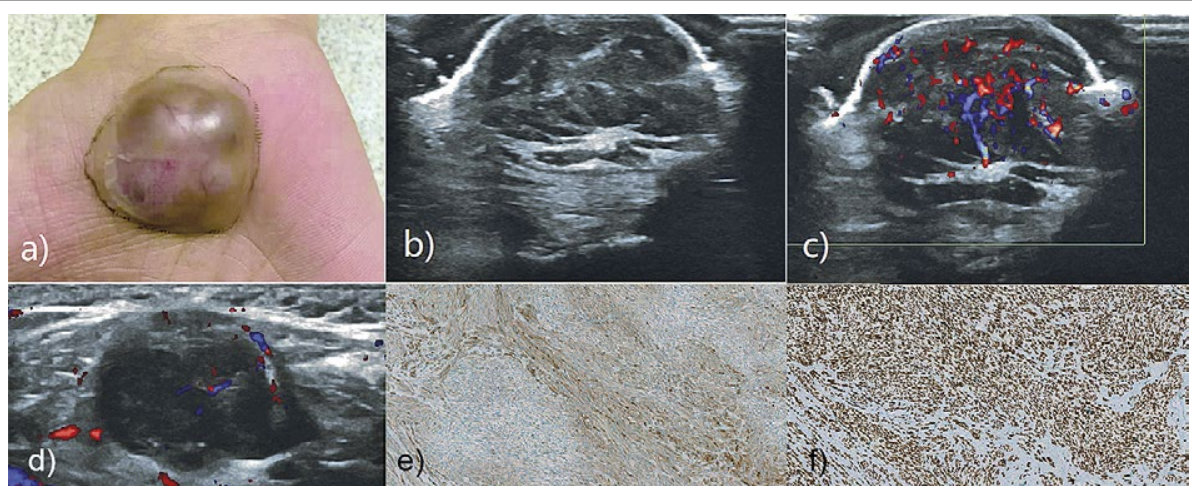

Fig 1. a) Clinical aspect of the mass on the right palm; b) B-mode ultrasound image of the mass on hand; c) color Doppler aspect of the mass on hand: d) color Doppler aspect of the right axillary mass; 1) immunohistochemistry showing S-100 (+); f) Immunohistochemistry showing SOX10 (+).

\section{References}

1. Thway K, Fisher C. Malignant peripheral nerve sheath tumor: pathology and genetics. Ann Diagn Pathol 2014;18:109-116.

2. Katz D, Lazar A, Lev D. Malignant peripheral nerve sheath tumour (MPNST): the clinical implications of cel- lular signalling pathways. Expert Rev Mol Med 2009;11: e30.

3. Sharma S, Shah JS, Bali H. Malignant peripheral nerve sheath tumor: A rare malignancy. J Oral Maxillofac Pathol 2020;24(Suppl 1):S86-S90.

4. Luzar B, Falconieri G. Cutaneous Malignant Peripheral Nerve Sheath Tumor. Surg Pathol Clin 2017;10:337-343.

\title{
Imaging findings of a metastatic myxopapillary ependymoma in the left inguinal region
}

\author{
Yunpeng Li ${ }^{1 *}$, Jiangfeng Wu ${ }^{1 *}$, Qingqing Fang ${ }^{2}$, Yun Jin ${ }^{1}$, Peiwen Wang ${ }^{1}$ \\ * the authors share the first authorship
}

${ }^{1}$ Department of Ultrasound, The Affiliated Dongyang Hospital of Wenzhou Medical University, Zhejiang, ${ }^{2}$ Department of Ultrasound, Tianxiang East Hospital, Yiwu, China

\section{To the Editor,}

A 21-year-old female presented to the general surgery department for a mass that had enlarged gradually in the left inguinal region in the last 5 years. At first, the mass was the size of a small walnut and now a fist. She de-

Received 22.07.2021 Accepted 05.10.2021

Med Ultrason

2021, Vol. 23, No 4, 495-496, DOI: 10.11152/mu-3339,

Corresponding author: Jiangfeng $\mathrm{Wu}$

Department of Ultrasound, The Affiliated

Dongyang Hospital of Wenzhou Medical

University, No. 60 Wuning West Road,

Dongyang 322100, Zhejiang, China

E-mail:wjfhospital@163.com

Phone: +8618257937213 nied tenderness, abdominal pain, as well as paresthesia of lower extremities and her family was negative for any history of malignancy. A physical examination showed no remarkable abnormality except for the mass. The patient underwent sacrococcygeal tumor resection 10 years ago, but she could not depict the histopathology of the tumor definitely.

Ultrasonographic examination revealed a homogeneous, solid and hyperechoic mass, $10.0 \times 7.4 \times 5.6 \mathrm{~cm}$, with clear boundary, oval in shape (fig 1a), and internal linear septations (fig 1b) in the left inguinal region. There were dot-like blood flow signals in the mass, as monitored by Doppler mode (fig 1c). MRI enhancement scan revealed the mass was uneven enhanced (fig 1d).

Histopathology reports confirmed the diagnosis of metastatic myxopapillary ependymoma (ME) that is con- 
sistent with the initial pathology of the primary sacrococcygeal tumor (fig 1e, f).

To our best knowledge, sacrococcygeal ME metastasizing to the groin has not been reported, while several studies demonstrated inguinal lymph node metastasis of ME $[1,2]$.

An extradural ME is considered as a subgroup ependymoma, which is often misdiagnosed and should be considered in the differential diagnosis of any soft tissue mass such as teratoma, neurogenic tumor, soft tissue sarcoma, metastatic carcinoma, pilonidal sinus, cyst and ME subtypes.

Our case of rarity demonstrates that the sacrococcygeal ME that has been resected may metastasize to the groin after many years. Ultrasound imaging plays an important role in the assessment of a soft tissue tumor, as is a non-invasive, radiation-free, available, and inexpensive tool. The present case might make for the comprehension of rare and late metastatic ME and be helpful in the diagnosis of the soft tissue tumors of unknown origin.

\title{
References
}

1. Vega-Orozco R, Rembao-Bojórquez D, Salmerón-Mercado M, García-Marquez A, Tena-Suck ML. Inguinal lymph nodal metastasis of myxopapillary ependymoma confirmed by fine-needle aspiration cytology, biopsy, and immunohistochemistry: case report. Diagn Cytopathol 2011;39:689-693.

2. Kramer GW, Rutten E, Sloof J. Subcutaneous sacrococcygeal ependymoma with inguinal lymph node metastasis. Case report. J Neurosurg 1988;68:474-477.

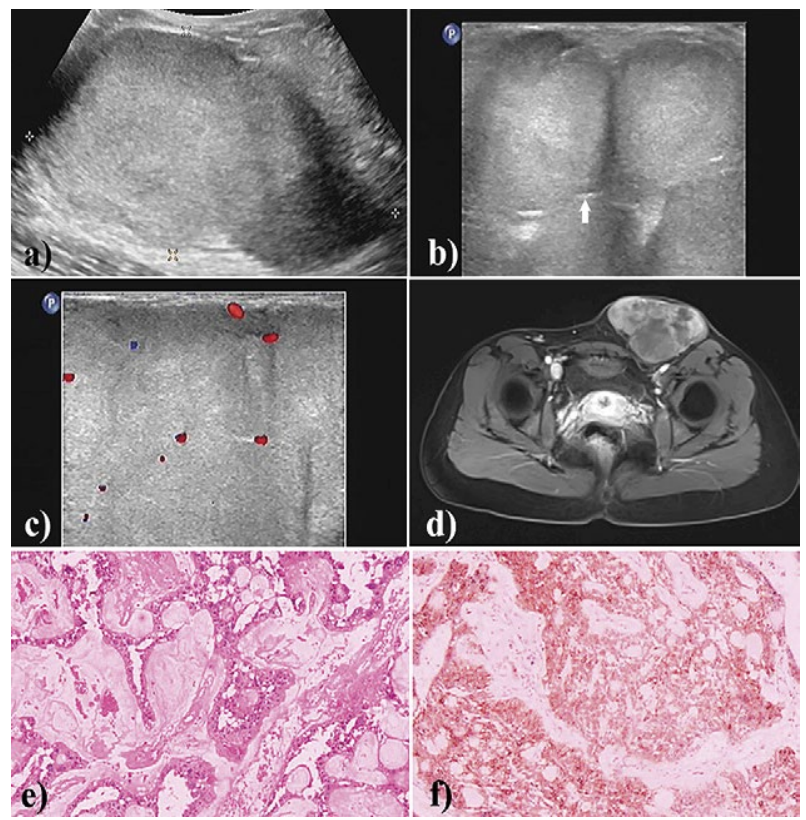

Fig 1. a) Ultrasound reveals a homogeneous, solid and hyperechoic mass with clear boundary and oval in shape; b) Doppler mode shows dot-like blood flow signals in the mass; c) internal septations of the mass are shown (arrow); d) MRI enhancement scan reveals the mass is uneven enhanced; e) hematoxylin and eosin sections of the mass demonstrates myxoid and vascularized stroma revealing papillary structures with single rowed cuboidal columnar epithelium and mucin within the lumen; f) immunohistochemical analysis reveals positive reactivity for glial fibrillary acidic protein (GFAP)

\section{Comment to: The utility of ultrasound examination in cubital tunnel syndrome caused by heterotopic ossification}

\author{
Yei Heum Park, Daeseok Oh
}

Department of Anesthesia and Pain Medicine, Inje University Haeundae Paik Hospital, Busan, Republic of Korea

Received 14.10.2021 Accepted 21.10.2021

Med Ultrason

2021, Vol. 23, No 4, 496-497, DOI: 10.11152/mu-3458,

Corresponding author: Daeseok Oh, MD

Department of Anesthesia \& Pain Medicine,

Inje University Haeundae Paik Hospital,

Busan, Republic of Korea,

875, Haeun-daero, Haeundae-gu, Busan,

612-896 Republic of Korea

Phone: 82-51-797-0415

Fax: 82-51-797-2669

E-mail: yivangin@naver.com

\section{To the Editor}

Ultrasound (US) can visualize the pathological anatomy of heterotopic ossification (HO) and the compression location of the nerve in the cubital tunnel [1]. We read with interest the article of Jačisko et al [2]. In addition, we report rare US images of HO in direct contact with the swollen ulnar nerve that was not detected by plain radiography. A 60-year-old female presented with a six-month history of elbow pain. Her pain was located 


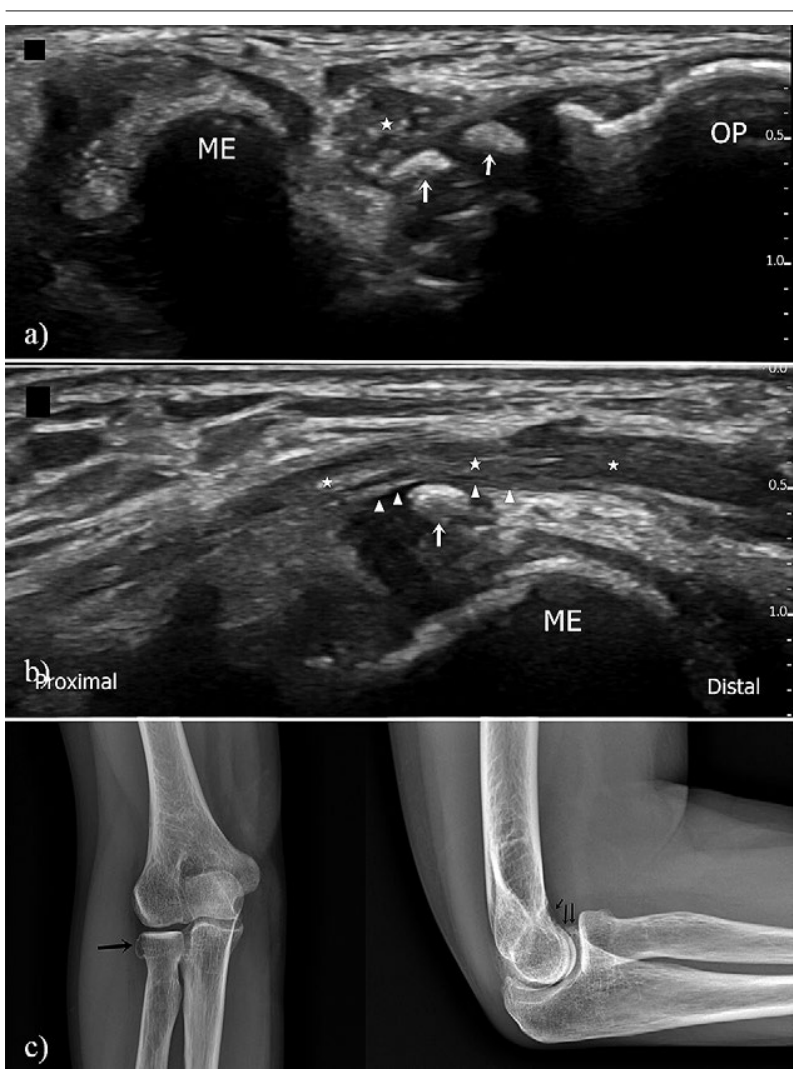

Fig 1. Ultrasonographic scan, transverse (a) and longitudinal view (b). Heterotopic ossification (arrows) causing acoustic shadowing, in contact with the ulnar nerve (arrowheads). Stars indicated the ulnar nerve. Radiography (c), black arrows indicate osteophyte formation. ME: medical epicondyle; OP: olecranon process.

at the medial side of the right elbow joint and accompanied by numbness of the fifth finger. US showed hyperechoic masses causing acoustic shadowing, in direct contact with the ulnar nerve in the cubital tunnel (fig la,b). Plain radiographs demonstrated osteophyte formation in the coronoid process of the ulna, the coronoid fossa of the humerus and in the radial head (fig 1c). Radiography showed no heterotopic bone formation in the soft tissues surrounding the medial side of the right elbow. We performed US-guided perineural injection with a mixture of $1 \mathrm{cc}$ of $10 \mathrm{mg}$ triamcinolone and $3 \mathrm{cc}$ of $0.2 \%$ ropivacaine. Her pain and numbness diminished with no adverse effects. Her pain reduced by $70 \%$ after two weeks, with pain improvement sustained for 6 months after the injection. Jačisko et al [2] have presented some diagnostic US on neuropathy caused by HO located close to the ulnar nerve in the cubital tunnel. Especially, this case showed definite $\mathrm{HO}$ in the soft tissue surrounding the medial side of the elbow on radiography. The classic US of $\mathrm{HO}$ was defined by the presence of a central hypoechoic area surrounded by calcification [3]. The distortion of normal soft tissue and the formation of hypoechoic areas, with or without foci of calcification can also be shown as early signs [3]. The use of US for HO is highly sensitive and provides an earlier diagnosis compared with other radiologic modalities [3]. It can be an effective treatment strategy and may improve the prognosis of neuropathy. We highlight that US can provide early diagnostic information about ulnar nerve morphology and various $\mathrm{HO}$ even if radiography does not show heterotopic bone formation.

\section{References}

1. Nakashian MN, Ireland D, Kane PM. Cubital Tunnel Syndrome: Current Concepts. Curr Rev Musculoskelet Med 2020;13:520-524.

2. Jačisko J, Sobotová K, Mezian K. The utility of ultrasound examination in cubital tunnel syndrome caused by heterotopic ossification. Med Ultrason 2020;22:117-118.

3. Stefanidis K, Brindley P, Ramnarine R, et al: Bedside U1trasound to Facilitate Early Diagnosis and Ease of FollowUp in Neurogenic Heterotopic Ossification: A Pilot Study From the Intensive Care Unit. J Head Trauma Rehabil 2017;32:E54-E58. 